\title{
La satisfacción corporal y su rol en las relaciones sociales
}

Body Satisfaction and its Role in Social Relations

Recibido: mayo 12 de 2011 | Revisado: marzo 1 de 2012 | Aceptado: julio 22 de 2012

\author{
Pedro Gil Madrona* \\ Universidad de Castilla-La Mancha, España
}

doi:10.11144/Javeriana.UPSY12-2.scrr

Para citar este artículo: Gil, P. (2013). La satisfacción corporal y su rol en las relaciones sociales. Universitas Psychologica, 12(2), 547-558.

* Profesor Titular de la Universidad de Castilla-La Mancha, España. Departamento de Didáctica de la Expresión Plástica, Musical y Corporal. E-mail: Pedro.Gil@uclm.es

\section{RES UMEN}

Se analiza el nivel de satisfacción de las personas con su propio cuerpo y la visión que estas tienen sobre el resto de la sociedad. Se trata de comprobar el nivel de satisfacción corporal y la visión sobre el propio cuerpo, conocer la visión que la sociedad tiene sobre el resto de personas y la forma de aceptación de las mismas, conocer por sexos a quien afecta más la preocupación por el físico y la visión y la opinión de la sociedad sobre la importancia de los medios de comunicación en la visión del propio cuerpo. La selección de la muestra se llevó a cabo de manera aleatoria, compuesta por 203 personas de la ciudad de Albacete (España) y que se categorizó y tabuló con base en las variables género, complexión física y edad (distinguiendo entre mayores y menores de 30 años). El cuestionario utilizado, surgido tras la lectura del artículo de Duncan (2007), constaba de un total de 22 preguntas de las que se recogieron para este artículo los datos de ocho de ellas. Tras el estudio, el cuerpo ha quedado definido como sede de la subjetividad y, en consecuencia, como punto de conexión útil para la conformación de un mundo social, en lo que se refiere al ámbito de la trayectoria que el sujeto adoptará a la largo de toda su vida.

Palabras clave autores

Cuerpo, interacción social, socialización, percepción, sensibilidad.

Palabras clave descriptores

Satisfacción personal, imagen corporal investigación cuantitativa.

\section{A B S T R A C T}

The research here tries to investigate the level of satisfaction of people with their own body and their view on the rest of society. In our article we analyze the role of body as a vehicle of socialization and, subsequently, of transmission of contents and discourses among society members. Firstly, we are trying to check the level of physical satisfaction and the view of the own body. Secondly, we will try to discover the viewpoint that society has of the rest of the people and the compatibility between those viewpoints. Thirdly, we will separate by sexes in order to investigate in a more detailed way the group of people that is affected by the worry about their own body. Lastly, the investigation will focus on the general opinion on the influence of the media amongst the view of the own physical image and the integration of people in several sports.

Key words authors

Body, social interaction, socialization, perception, sensitivity.

Key words plus

Personal Satisfaction, Self-corporal Image, Quantitative Research. 


\section{Introducción}

El estudio centra la atención en el cuerpo, un particular tipo de materia que tiene como característica intrínseca y altamente definitoria, la posibilidad de pensamiento, sentimiento y sensibilidad. En el análisis, se asume tanto la perspectiva que Crossley (1995) considera propia de la "sociología del cuerpo", como la de lo que denomina "sociología carnal". Mientras que la primera presta atención a lo que se le hace al cuerpo, la segunda se centra en lo que el cuerpo hace. Esta doble dualidad es asumible porque la doble dirección resulta necesaria para una adecuada percepción de las implicaciones del cuerpo en la vida social. Este trabajo se distancia, en consecuencia, de las posiciones que tienden a elaborar teorías de lo social que ignoran el cuerpo $-\mathrm{y}$, a la inversa, teorías del cuerpo para las que no existe lo social-; se planteará su indisociable vínculo para la comprensión de la realidad social, como ya se reclamara-un tanto minoritariamente-desde la sociología clásica (Freud, 1961; Giddens, 1984; Goffman, 1959, 1961, 1963, 1979).

De igual forma se presta especial atención a las especiales aportaciones de la fenomenología del cuerpo (Husserl, 1997; Merleau-Ponty, 2003; Todes, 2001) para quienes en el cuerpo se cruzan realidad y vivencia. El cuerpo es la realidad subjetiva en que comparece la realidad de las otras cosas y seres, desde el movimiento a las actividades, desde las manipulaciones a las relaciones sociales, desde la interacción con las cosas, los estados afectivos y desde las capacidades lingüísticas y expresivas a los juegos y las danzas (San Martín, 2010). A tal efecto, la fenomenología expresa dos modos del cuerpo. Por una parte, el cuerpo es considerado como cuerpo objeto y, por otra parte, el cuerpo es considerado como cuerpo propio, cuerpo vivido y cuerpo animado. La corporalidad se presenta, por tanto, como ser-cuerpo y tener-cuerpo (Runge, 2004). Pues el descubrimiento del cuerpo como organismo material por las ciencias naturales, como la medicina o la genética, vino acompañado del redescubrimiento del cuerpo como realidad radical de la vida humana y de la cultura en las Ciencias Humanas.
Ciertamente, se asume que, como señala Connell (1995, p. 50) con un juego de palabras muy significativo, "los cuerpos, en su propio derecho como tales, importan". La clave radica en determinar a través de qué medios las personas, como seres humanos provistos de cuerpos igualmente humanos, pueden establecer el vínculo entre lo material y lo social con la mediación de los cuerpos.

El análisis de cómo acontece esto ha sido señalado tanto en lo referente al género humano en su conjunto como en lo que concierne a un individuo determinado. En la primera acepción, se ha observado que la propia especie humana como tal está sometida a un permanente proceso de reconstrucción corporal en el que es fácil rastrear la influencia de lo social. En este sentido, Salinas (1994, p. 96) ha apreciado que "la influencia de los factores sociales tales como la economía, la educación, la religión, la profesión, etc., no tendría ningún efecto sobre los procesos de construcción de la identidad del individuo a menos que procesos interactivos (el proceso cognitivo) y estructuras específicas (las funciones cerebrales) favorezcan todo tipo de interacciones entre la realidad 'externa' (...) y el propio 'sujeto'. (...). Las características funcionales del cuerpo humano (...) habrían sido determinadas a lo largo de millones de años de evolución de la cultura social manifestada en los procesos de humanización".

La consecuencia de todo ese amplio flujo de condicionantes sociales sobre cada una de las personas como individuos es algo que ha sido expresado de una manera muy intuitiva por Scarry (1985, p. 109) al escribir que "cuando un hombre se mueve a través de la habitación, a través de su hombro, cabeza, caderas, piernas y brazos irradia la historia de los años de su primera infancia en Georgia y su adolescencia juvenil en Manhattan”. A primera vista, muy probablemente esta frase se considerará excesivamente exagerada, porque hace referencia únicamente a movimientos corporales; la influencia de lo social no podría eclipsar tan radicalmente el potencial altamente condicionante de lo que puede ser pensado como puramente físico -es decir, objetivo o material-. Pero podemos considerar, además, aspectos corporales que, más allá de puros 
movimientos, son asimismo signos indicativos de la influencia de lo social.

De esta manera, las formas que adopta la conducta corporal -los gestos, la postura, las maneras, los estilos de vestir- pueden ser consideradas como una forma de transmitir o comunicar ciertos significados en mundos sociales diferenciados. La conducta corporal es así, a la vez, mediada por y, también, como pone de relieve el profesor Miranda (2002, pp. 73-74), mediadora de lo social: "El mundo de significado que yo he ido construyendo desde mi nacimiento, y en el que habito, se me ha ido dando (...) interpretado lingüísticamente a través de los gestos, de las caricias, de las sonrisas, de los besos, de las lágrimas y de los relatos (...) de todos aquellos cuerpos que se me han hecho y se me hacen presentes".

Este mecanismo es expresado en términos sociológicos a través del concepto de habitus. Normalmente asociado a los fértiles trabajos de Bourdieu (1998), aunque el concepto como tal tiene su origen en las observaciones de Mauss (1973, p. 88), que lo diferenciaba de lo que pudiera considerarse como un mero hábito: "Estos hábitos no varían solo en función de los individuos (...); varían especialmente entre sociedades, educaciones, propiedades y modas (...)".

Para Bourdieu (1998, p. 202), el habitus es la expresión de un mundo social internalizado. En este sentido podemos ver unos datos especialmente significativos al respecto, que nos ofrece en su conocida obra La distinción: criterio y bases sociales del gusto. En ella, observa como el gusto, las preferencias estéticas y, en consecuencia, las pautas de consumo son afectadas por las estructuras sociales a través del habitus (véanse las variaciones en el valor otorgado al cuerpo, la belleza, al gusto sexual en función de la imagen corporal, al maquillaje y el cuidado corporal como es el bañarse o el acudir al peluquero en función de si el cabeza de familia es un agricultor o un obrero cuyo estatus social califica de bajo, un empleado de estatus social calificado de medio o un industrial o profesional liberal categorizado como de estatus social superior). En dicho trabajo se puede percibir como el cuerpo es mucho más que un trozo de materia puramente físico; por el contrario, es considerado como una entidad social. De esta manera, cobra pleno sentido el concepto de habitus de Bourdieu (1998), ya que según la definición propuesta por Nash (1999, p. 177), para quien "el habitus es concebido [por Bourdieu] como un esquema generativo a través del cual las formas de las estructuras sociales básicas adquieren corporeidad en los individuos, con el resultado de que la gente actúa necesariamente de tal manera que las estructuras subyacentes son reproducidas cobrando efecto".

Una traducción comercial de esta realidad en las generaciones más juveniles se ha observado en el uso que los editores de revistas femeninas para ese tramo de edad hacen de la imagen corporal de las chicas. En efecto, Ciudad (2001) ofrece un contraste entre las imágenes del cuerpo en revistas de este tipo con costes, estéticas y públicos muy diferenciados. La diferenciación la establece entre unas publicaciones de coste económico comparativamente elevado, editadas en papel couché y con contenidos muy asociados a figuras muy características (Enrique Iglesias, Sakira, Alejandro Sanz) del panorama musical, y otras más populares, de coste económico más reducido, cuya calidad de materiales es más normalizada y con presencia de figuras (como es el caso del dúo musical Estopa) de connotaciones distintas a las habituales en la primera. Las características corporales que se observan en la publicidad de las revistas del primer tipo son, sin duda, más sofisticadas que las que encontramos en el tipo más "popular", como lo son también las "técnicas" de realce del cuerpo. Las representaciones corporales en el segundo tipo cobran un protagonismo más reducido que en el tipo, digamos, más "sofisticado", y las connotaciones de aquella están marcadas por una mayor naturalidad que se corresponde -de nuevo, muy en la línea bourdieuana- con los pautas de consumo y preferencias estéticas del público de la revista. La conexión entre estas, de un lado, y valor y concepto del cuerpo, de otro, se ponen de manifiesto, pues, a través de este contraste.

Desde mediados del siglo XX, el cuerpo es tema prioritario de la ideología del consumismo, centrada en el culto al cuerpo bello y el interés por la salud. Ese culto al cuerpo, desde la ideología del consu- 
mismo nos presiona psicológicamente y socialmente para gastar energía, tiempo y dinero, en el estéril y frustrante intento de alcanzar la figura ideal, el cuerpo perfecto (Díaz Rojo, 2006).

No se puede pasar por alto, a su vez, lo que se ha dado en llamar la revolución somatoplástica para entender las intrincadas interacciones entre ciencias tecnológicas de la vida y las nuevas pautas culturales respecto al cuerpo. Bajo esta idea, el cuerpo humano es una realidad plástica modificable, de tal manera que se puede controlar y modificar el cuerpo, en muy diversos aspectos, por los medios tecnológicos y científicos disponibles (dietética, cirugía plástica, farmacia, cosmética, educación física y deportiva, etc.) según diversos criterios de salud, belleza, bienestar o éxito social. Estos criterios, están indicando, a su vez, nuevas realidades sociales y nuevas necesidades sociales relativos a la arquetípica imagen del propio cuerpo (Ruiz Calvete, 2010). Dichas necesidades se imponen a millones de personas en todo el planeta a través de los medios de comunicación de masas. En efecto, los medios de comunicación de masas están siendo responsables, en los últimos diez años, más o menos, del desmesurado aumento del público en general por interesarse por la salud y la forma física (Rysst, 2010). A tal efecto, se está produciendo un aumento de diversas prácticas corporales como dieta, maquillaje, cosmética, la cirugía y el interés por la ropa. Esto ha llevado a Jones (2008) a decir que se está produciendo un cambio de imagen cultural.

En definitiva, vemos que no resulta difícil levantar la vista de nuestra actitud natural -en la que habitualmente no nos percatamos de estas cosas- (Schutz, 1967, p. 208) y apreciar como los cuerpos se comportan socialmente como algo más que trozos de realidad material. La cotidianeidad de estos usos del cuerpo se pone de relieve en el reciente titular de prensa del diario El País, que muy expresivamente proclama que "El mensaje está en el cuerpo" (Sánchez-Vallejo, 2009, p. 32).

La referencia al cuerpo como medium de transmisión de mensajes y como reflejo del mundo social en que se inscribe la vida de los individuos no va en detrimento de sus potencialidades como elemento clave para definir jerarquías sociales. Shilling (1991, p. 118) parafrasea a Pierre Bourdieu -en concreto, su valioso concepto "capital cultural" -y propone considerar el rol de lo que denomina "capital físico". Es decir, yendo un paso más allá que Pierre Bourdieu, Shilling plantea el valorar la manera en que el cuerpo determina las actividades en las que el individuo tendrá mayor o menor éxito social o profesional. Si el capital cultural mostraba un fuerte valor condicionante del desarrollo de las personas en el seno social, lo mismo ocurre con el capital físico que reside en el cuerpo. En la misma línea, en un artículo anterior trata de exponer la forma en que el cuerpo-o, lo que es lo mismo, el capital físico-actúa como factor condicionante del establecimiento de las desigualdades sociales.

Se debe prestar atención también a la teoría de la cognición a través del cuerpo, que se fundamenta en que la cognición está relacionada con las interacciones del cuerpo con el medio ambiente, si bien al no ser objeto de este estudio queda apartado para abordarlo en otra ocasión.

\section{Método}

La investigación intenta conocer, de un lado, el nivel de satisfacción corporal y la visión sobre el propio cuerpo que tienen las personas. Junto a esto, se ha pretendido conocer la visión que la sociedad tiene sobre el resto de personas y las formas de aceptación o rechazo de las mismas con base en aspectos corporales. En tercer lugar, se pretende ver de manera más detallada -con diferenciación de sexo y edad- a quién afecta más la preocupación por el físico y la visión que del mismo tienen las personas. Y, por último, la investigación se ha centrado en conocer la opinión que la sociedad tiene sobre el consumo y la dieta para mejorar la visión corporal.

\section{Participantes}

La selección de la muestra se llevó a cabo de manera aleatoria y estuvo compuesta por 203 sujetos. Los datos se han categorizado y tabulado con base en las variables género, complexión física y edad (dis- 
tinguiendo en este caso, entre mayores y menores de 30 años).

\section{Instrumento}

El cuestionario utilizado, surgido tras la lectura del artículo de Duncan (2007), consta de un total de 22 preguntas de las que se recogieron para este artículo los datos de ocho de ellas, claramente orientadas al planteamiento teórico de este texto y que, en consecuencia, dan respuesta a los objetivos del estudio.

\section{Resultados}

Inquietud por el cuidado de la imagen corporal

Se pretende verificar con datos en qué medida existía un condicionamiento social en lo que se refiere al cuidado de la imagen corporal como consecuencia de que la persona es observada socialmente. En este sentido, una primera cuestión que se planteó fue, genéricamente, la recogida en el

TABLA 1

Tabla de contingencia Complexión y Género: ¿Cuidas tu imagen corporal por el hecho de que sabes que el resto del mundo te mira?

\begin{tabular}{|c|c|c|c|c|c|c|c|c|}
\hline & \multirow{2}{*}{ Complexión } & \multicolumn{7}{|c|}{ Ítem 1} \\
\hline & & Nada & Muy poco & Algo & A veces & Bastante & Mucho & Total \\
\hline \multirow{4}{*}{ Delgado } & Recuento & 1 & 3 & 6 & 5 & 3 & 0 & 18 \\
\hline & \% dentro de Compl. & 5.6 & 16.7 & 33.3 & 27.8 & 16.7 & 0.0 & 100 \\
\hline & $\%$ dentro de 6 & 2.1 & 6.7 & 13.3 & 11.6 & 17.6 & 0.0 & 8.9 \\
\hline & $\%$ del total & 0.5 & 1.5 & 3 & 2.5 & 1.5 & 0.0 & 8.9 \\
\hline \multirow{4}{*}{ Normal } & Recuento & 36 & 31 & 29 & 32 & 12 & 3 & 143 \\
\hline & \% dentro de Compl. & 25.2 & 21.7 & 20.3 & 22.4 & 8.4 & 2.1 & 100 \\
\hline & $\%$ dentro de 6 & 75 & 68.9 & 64.4 & 74.4 & 70.6 & 60 & 70.4 \\
\hline & $\%$ del total & 17.7 & 15.3 & 14.3 & 15.8 & 5.9 & 1.5 & 70.4 \\
\hline \multirow{4}{*}{$\begin{array}{l}\text { Sobre- } \\
\text { peso }\end{array}$} & Recuento & 11 & 11 & 10 & 6 & 2 & 2 & 42 \\
\hline & \% dentro de Compl. & 26.2 & 26.2 & 23.8 & 14.3 & 4.8 & 4.8 & 100 \\
\hline & $\%$ dentro de 6 & 22.9 & 24.4 & 22.2 & 14 & 11.8 & 40 & 20.7 \\
\hline & $\%$ del total & 5.4 & 5.4 & 4.9 & 3.0 & 1.0 & 1.0 & 20.7 \\
\hline \multirow{4}{*}{ Total } & Recuento & 48 & 45 & 45 & 43 & 17 & 5 & 203 \\
\hline & \% dentro de Compl. & 23.6 & 22.2 & 22.2 & 21.2 & 8.4 & 2.5 & 100 \\
\hline & $\%$ dentro de 6 & 100 & 100 & 100 & 100 & 100 & 100 & 100 \\
\hline & $\%$ del total & 23.6 & 22.2 & 22.2 & 21.2 & 8.4 & 2.5 & 100 \\
\hline & Género & Nada & Muy poco & Algo & A veces & Bastante & Mucho & Total \\
\hline \multirow[t]{4}{*}{ Hombre } & Recuento & 16 & 18 & 23 & 24 & 7 & 4 & 92 \\
\hline & $\%$ dentro de Género & 17.4 & 19.6 & 25 & 26.1 & 7.6 & 4.3 & 100 \\
\hline & $\%$ dentro de 6 & 33.3 & 40 & 51.1 & 55.8 & 41.2 & 80 & 45.3 \\
\hline & $\%$ del total & 7.9 & 8.9 & 11.3 & 11.8 & 3.4 & 2 & 45.3 \\
\hline \multirow[t]{4}{*}{ Mujer } & Recuento & 32 & 27 & 22 & 19 & 10 & 1 & 111 \\
\hline & $\%$ dentro de Género & 28.8 & 24.3 & 19.8 & 17.1 & 9 & 0.9 & 100 \\
\hline & $\%$ dentro de 6 & 66.7 & 60 & 48.9 & 44.2 & 58.8 & 20 & 54.7 \\
\hline & $\%$ del total & 15.8 & 13.3 & 10.8 & 9.4 & 4.9 & 0.5 & 54.7 \\
\hline \multirow[t]{4}{*}{ Total } & Recuento & 48 & 45 & 45 & 43 & 17 & 5 & 203 \\
\hline & $\%$ dentro de Género & 23.6 & 22.2 & 22.2 & 21.2 & 8.4 & 2.5 & 100 \\
\hline & $\%$ dentro de 6 & 100 & 100 & 100 & 100 & 100 & 100 & 100 \\
\hline & $\%$ del total & 23.6 & 22.2 & 22.2 & 21.2 & 8.4 & 2.5 & 100 \\
\hline
\end{tabular}

Fuente: elaboración propia. 
TABLA 2

Tabla de contingencia Género. ¿Quiénes piensas que pueden estar más afectados por este hecho, los hombres o las mujeres?

\begin{tabular}{|c|c|c|c|c|c|}
\hline & & & \multicolumn{3}{|c|}{ Ítem 2} \\
\hline & & & Hombre & Mujer & Total \\
\hline \multirow{8}{*}{ Género } & \multirow{4}{*}{ Hombre } & Recuento & 2 & 90 & 92 \\
\hline & & $\%$ dentro de Género & 2.2 & 97.8 & 100 \\
\hline & & $\%$ dentro de 8 & 33.3 & 45.7 & 45.3 \\
\hline & & $\%$ del total & 1 & 44.3 & 45.3 \\
\hline & \multirow{4}{*}{ Mujer } & Recuento & 4 & 107 & 111 \\
\hline & & $\%$ dentro de Género & 3.6 & 96.4 & 100 \\
\hline & & $\%$ dentro de 8 & 66.7 & 54.3 & 54.7 \\
\hline & & $\%$ del total & 2 & 52.7 & 54.7 \\
\hline \multirow{4}{*}{ Total } & & Recuento & 6 & 197 & 203 \\
\hline & & \% dentro de Género & 3 & 97 & 100 \\
\hline & & $\%$ dentro de 8 & 100 & 100 & 100 \\
\hline & & $\%$ del total & 3 & 97 & 100 \\
\hline
\end{tabular}

Fuente: elaboración propia.

ítem 1: "icuidas tu imagen corporal por el hecho de que sabes que el resto del mundo te observa?". Las respuestas a esta cuestión, distribuidas en función de la complexión física de los entrevistados (Tabla 1), muestran cómo las categorías de respuesta nada o muy poco incrementan sus porcentajes a medida que aumenta el grado de la complexión, a la inversa de lo que ocurre con la categoría bastante. Las diferencias, aunque significativas, no deben ser sobredimensionadas, y parecen sugerir que, en cierto modo, las personas con mayor nivel de masa corporal tienden a expresar con mayor facilidad

TABLA 3

Tabla de contingencia Complexión. ¿Piensas que las personas con sobrepeso son víctimas de sus propios actos?

\begin{tabular}{|c|c|c|c|c|c|c|c|c|}
\hline & \multirow[b]{2}{*}{ Complexión } & \multicolumn{6}{|c|}{ Ítem 6} & \multirow{2}{*}{ Tota } \\
\hline & & Nada & Muy poco & Algo & A veces & Bastante & Mucho & \\
\hline \multirow{4}{*}{ Delgado } & Recuento & 2 & 2 & 2 & 3 & 5 & 4 & 18 \\
\hline & \% dentro de Compl. & 11.1 & 11.1 & 11.1 & 16.7 & 27.8 & 22.2 & 100 \\
\hline & $\%$ dentro de 15 & 4.8 & 4.1 & 7.7 & 6.0 & 20.0 & 36.4 & 8.9 \\
\hline & $\%$ del total & 1 & 1 & 1 & 1.5 & 2.5 & 2 & 8.9 \\
\hline \multirow{4}{*}{ Normal } & Recuento & 18 & 34 & 18 & 46 & 20 & 7 & 143 \\
\hline & $\%$ dentro de Compl. & 12.6 & 23.8 & 12.6 & 32.2 & 14.0 & 4.9 & 100 \\
\hline & $\%$ dentro de 15 & 42.9 & 69.4 & 69.2 & 92.0 & 80.0 & 63.6 & 70.4 \\
\hline & $\%$ del total & 8.9 & 16.7 & 8.9 & 22.7 & 9.9 & 3.4 & 70.4 \\
\hline \multirow{8}{*}{ Sobrepeso } & Recuento & 22 & 13 & 6 & 1 & 0 & 0 & 42 \\
\hline & \% dentro de Compl. & 52.4 & 31 & 14.3 & 2.4 & 0.0 & 0.0 & 100 \\
\hline & $\%$ dentro de 15 & 52.4 & 26.5 & 23.1 & 2 & 0.0 & 0.0 & 20.7 \\
\hline & $\%$ del total & 10.8 & 6.4 & 3.0 & 0.5 & 0.0 & 0.0 & 20.7 \\
\hline & Recuento & 42 & 49 & 26 & 50 & 25 & 11 & 203 \\
\hline & \% dentro de Compl. & 20.7 & 24.1 & 12.8 & 24.6 & 12.3 & 5.4 & 100 \\
\hline & $\%$ dentro de 15 & 100 & 100 & 100 & 100 & 100 & 100 & 100 \\
\hline & $\%$ del total & 20.7 & 24.1 & 12.8 & 24.6 & 12.3 & 5.4 & 100 \\
\hline
\end{tabular}

Fuente: elaboración propia. 
TABLA 4

Tabla de contingencia Complexión. ¿Piensas, desde el sentido común, que el estar gordo es claramente algo equivocado?

\begin{tabular}{|c|c|c|c|c|c|c|c|c|}
\hline & \multicolumn{8}{|c|}{ Ítem 7} \\
\hline & Complexión & Nada & Muy poco & Algo & A veces & Bastante & Mucho & Total \\
\hline \multirow{4}{*}{ Delgado } & Recuento & 4 & 3 & 1 & 4 & 4 & 2 & 18 \\
\hline & \% dentro de Compl. & 22.2 & 16.7 & 5.6 & 22.2 & 22.2 & 11.1 & 100 \\
\hline & $\%$ dentro de 16 & 5 & 8.8 & 4.3 & 10.5 & 16 & 66.7 & 8.9 \\
\hline & $\%$ del total & 2 & 1.5 & 0.5 & 2 & 2 & 1 & 8.9 \\
\hline \multirow{4}{*}{ Normal } & Recuento & 39 & 28 & 22 & 32 & 21 & 1 & 143 \\
\hline & \% dentro de Compl. & 27.3 & 19.6 & 15.4 & 22.4 & 14.7 & 0.7 & 100 \\
\hline & $\%$ dentro de 16 & 48.8 & 82.4 & 95.7 & 84.2 & 84.0 & 33.3 & 70.4 \\
\hline & $\%$ del total & 19.2 & 13.8 & 10.8 & 15.8 & 10.3 & 0.5 & 70.4 \\
\hline \multirow{4}{*}{ Sobrepeso } & Recuento & 37 & 3 & 0 & 2 & 0 & 0 & 42 \\
\hline & \% dentro de Compl. & 88.1 & 7.1 & 0.0 & 4.8 & 0.0 & 0.0 & 100 \\
\hline & $\%$ dentro de 16 & 46.3 & 8.8 & 0.0 & 5.3 & 0.0 & 0.0 & 20.7 \\
\hline & $\%$ del total & 18.2 & 1.5 & 0.0 & 1 & 0.0 & 0.0 & 20.7 \\
\hline \multirow{4}{*}{ Total } & Recuento & 80 & 34 & 23 & 38 & 25 & 3 & 203 \\
\hline & \% dentro de Compl. & 39.4 & 16.7 & 11.3 & 18.7 & 12.3 & 1.5 & 100 \\
\hline & $\%$ dentro de 16 & 100 & 100 & 100 & 100 & 100 & 100 & 100 \\
\hline & $\%$ del total & 39.4 & 16.7 & 11.3 & 18.7 & 12.3 & 1.5 & 100 \\
\hline
\end{tabular}

Fuente: elaboración propia.

en una encuesta su insatisfacción con el cuidado corporal que llevan a cabo.

Como puede apreciarse, en la pregunta no existe ningún contenido que indique una comparación entre varones y mujeres -un matiz que sí se introdujo, como veremos, en la pregunta 2-. Analizando los datos, se advierte que, con esta formulación de la pregunta, no aparecen diferencias entre ambos géneros que vayan más allá del entorno de los cinco puntos porcentuales en cada una de las categorías. Parece que, efectivamente, tanto hombres como mujeres asumen en medidas similares que se practica un cierto cuidado corporal consecuencia de que, inevitablemente, alguien nos va a mirar y debemos causar

\section{TABLA 5}

Tabla de contingencia Edad: ¿Crees que la gente busca el cuerpo perfecto para no ser centro de una crítica social?

\begin{tabular}{llccccccc}
\hline & \multicolumn{1}{c}{ Ítem 3 } \\
\cline { 2 - 8 } Edad & Nada & Muy poco & Algo & A veces & Bastante & Mucho & Total \\
\hline \multirow{4}{*}{+ de 30 } & Recuento & 3 & 5 & 8 & 18 & 5 & 0 & 39 \\
& \% dentro de Edad & 7.7 & 12.8 & 20.5 & 46.2 & 12.8 & 0.0 & 100 \\
& \% dentro de 11 & 25 & 17.9 & 21.6 & 25 & 12.8 & 0.0 & 19.2 \\
& \% del total & 1.5 & 2.5 & 3.9 & 8.9 & 2.5 & 0.0 & 19.2 \\
\hline \multirow{5}{*}{ - de 30 } & Recuento & 9 & 23 & 29 & 54 & 34 & 15 & 164 \\
& \% dentro de Edad & 5.5 & 14 & 17.7 & 32.9 & 20.7 & 9.1 & 100 \\
& \% dentro de 11 & 75 & 82.1 & 78.4 & 75 & 87.2 & 100 & 80.8 \\
& \% del total & 4.4 & 11.3 & 14.3 & 26.6 & 16.7 & 7.4 & 80.8 \\
\hline \multirow{3}{*}{ Total } & Recuento & 12 & 28 & 37 & 72 & 39 & 15 & 203 \\
& \% dentro de Edad & 5.9 & 13.8 & 18.2 & 35.5 & 19.2 & 7.4 & 100 \\
& \% dentro de 11 & 100 & 100 & 100 & 100 & 100 & 100 & 100 \\
& \% del total & 5.9 & 13.8 & 18.2 & 35.5 & 19.2 & 7.4 & 100 \\
\hline
\end{tabular}

Fuente: elaboración propia. 


\section{TABLA 6}

Tabla de contingencia Edad y Género: ¿Ves a la sociedad como un consumidor de las variantes de la actividad física, sean estas las ropas, la cirugía plástica, entrenamiento, suplementos, juegos de realidad virtual, etc.?

\begin{tabular}{clcccccc}
\hline & & \multicolumn{1}{c}{ Ítem 4 } & & & \\
\hline \multicolumn{1}{c}{ Edad } & & Muy poco & Algo & A veces & Bastante & Mucho & Total \\
\hline+ de 30 & Recuento & 6 & 10 & 17 & 5 & 1 & 39 \\
& \% dentro de Edad & 15.4 & 25.6 & 43.6 & 12.8 & 2.6 & 100 \\
& \% dentro de 12 & 33.3 & 25 & 28.3 & 7.6 & 5.3 & 19.2 \\
& \% del total & 3 & 4.9 & 8.4 & 2.5 & 0.5 & 19.2 \\
\hline - de 30 & Recuento & 12 & 30 & 43 & 61 & 18 & 164 \\
& \% dentro de Edad & 7.3 & 18.3 & 26.2 & 37.2 & 11 & 100 \\
& \% dentro de 12 & 66.7 & 75 & 71.7 & 92.4 & 94.7 & 80.8 \\
& \% del total & 5.9 & 14.8 & 21.2 & 30 & 8.9 & 80.8 \\
\hline Total & Recuento & 18 & 40 & 60 & 66 & 19 & 203 \\
& \% dentro de Edad & 8.9 & 19.7 & 29.6 & 32.5 & 9.4 & 100 \\
& \% dentro de 12 & 100 & 100 & 100 & 100 & 100 & 100 \\
& \% del total & 8.9 & 19.7 & 29.6 & 32.5 & 9.4 & 100 \\
\hline Género & & Muy poco & Algo & A veces & Bastante & Mucho & Total \\
\hline Hombre & Recuento & 4 & 12 & 23 & 43 & 10 & 92 \\
& \% dentro de Género & 4.3 & 3 & 25 & 46.7 & 10.9 & 100 \\
& \% dentro de 12 & 22.2 & 30 & 38.3 & 65.2 & 52.6 & 45.3 \\
& \% del total & 2.0 & 5.9 & 11.3 & 21.2 & 4.9 & 45.3 \\
\hline Mujer & Recuento & 14 & 28 & 37 & 23 & 9 & 111 \\
& \% dentro de Género & 12.6 & 25.2 & 33.3 & 20.7 & 8.1 & 100 \\
& \% dentro de 12 & 77.8 & 70 & 61.7 & 34.8 & 47.4 & 54.7 \\
& \% del total & 6.9 & 13.8 & 18.2 & 11.3 & 4.4 & 54.7 \\
\hline Total & Recuento & 18 & 40 & 60 & 66 & 19 & 203 \\
& \% dentro de Género & 8.9 & 19.7 & 29.6 & 32.5 & 9.4 & 100 \\
& \% dentro de 12 & 100 & 100 & 100 & 100 & 100 & 100 \\
& \% del total & 8.9 & 19.7 & 29.6 & 32.5 & 9.4 & 100 \\
\hline
\end{tabular}

Fuente: elaboración propia.

la mejor impresión posible. En la pregunta 2 se pide al entrevistado, explícitamente, que indique si cree que el hecho del cuidado corporal afecta en mayor medida a uno u otro género. Las cifras de la Tabla 2 no dejan lugar a dudas, en cuanto casi la totalidad de entrevistados apreciaron que era la mujer la que, en mayor medida que el varón, aparece afectada. Las diferencias en este sentido fueron prácticamente inapreciables entre los encuestados varones y mujeres.

Visión social y nivel de aceptación en función de su imagen corporal

Dentro del conjunto de cuestiones planteadas aparecen las esperadas diferencias en función de la llamada masa corporal, pero la opinión que se observó cuando se preguntaba a los encuestados acerca de si las personas con sobrepeso son víctimas de sus propios actos (ítem 6), resultó ser especialmente significativa (Tabla 3). Una respuesta afirmativa parecería indicar que, en mayor o menor medida, la situación de sobrepeso es fruto del propio comportamiento de la persona que lo padece, probablemente por falta de una actividad física o dieta adecuadas. Las opciones de respuesta mucho o bastante no recogen ni una sola elección por parte de los encuestados con sobrepeso, lo que parece indicativo de una percepción de la situación como algo que no han conseguido evitar; en la misma lógica, los porcentajes de respuestas nada o muy 
TABLA 7

Tabla de contingencia Edad: ¿Tienes algún tipo de rechazo hacia las personas cuyo cuerpo se aparta de la norma social?

\begin{tabular}{clccccc}
\hline & & \multicolumn{2}{c}{ Ítem $\mathbf{5}$} \\
\hline \multicolumn{1}{c}{ Edad } & Nada & Muy poco & Algo & A veces & Total \\
\hline \multirow{5}{*}{+ de 30 30} & 30 & 8 & 1 & 0 & 39 \\
& Recuento & 76.9 & 20.5 & 2.6 & 0.0 & 100 \\
& \% dentro de Edad & 17.9 & 25.8 & 50 & 0.0 & 19.2 \\
& \% dentro de 14 & 14.8 & 3.9 & 0.5 & 0.0 & 19.2 \\
\hline \multirow{5}{*}{ - de del total 30} & 138 & 23 & 1 & 2 & 164 \\
& Recuento & 84.1 & 14 & 0.6 & 1.2 & 100 \\
& \% dentro de Edad & 82.1 & 74.2 & 50 & 100 & 80.8 \\
& \% dentro de 14 & 68 & 11.3 & 0.5 & 1 & 80.8 \\
\hline \multirow{3}{*}{ Total } & \% del total & 168 & 31 & 2 & 2 & 203 \\
& Recuento & 82.8 & 15.3 & 1 & 1 & 100 \\
& \% dentro de Edad & 100 & 100 & 100 & 100 & 100 \\
& \% dentro de 14 & 82.8 & 15.3 & 1 & 1 & 100 \\
\hline
\end{tabular}

Fuente: elaboración propia.

poco son mayoritariamente escogidas por este grupo de encuestados (que suman entre ambas un 83.4) -desde luego, muy por encima de las elecciones de las personas de complexión normal o delgada-.
En una línea coincidente, se quiso conocer el punto de vista de los entrevistados acerca de en qué medida consideraban, más allá de informes científicos o médicos -es decir, con base en el "sentido

\section{TABLA 8}

Tabla de contingencia Complexión: iPiensas que las personas con sobrepeso se obligan a seguir una dieta equilibrada para intentar superar sus problemas y evitar que la gente las observe?

\begin{tabular}{llccccccc}
\hline & \multicolumn{1}{c}{ Ćtem 8 } \\
\cline { 2 - 8 } \multicolumn{1}{c}{ Complexión } & Nada & $\begin{array}{c}\text { Muy } \\
\text { poco }\end{array}$ & Algo & A veces & Bastante & Mucho & \multirow{2}{*}{ Total } \\
\hline \multirow{4}{*}{ Delgado } & Recuento & 0 & 0 & 2 & 6 & 5 & 5 & 18 \\
& \% dentro de Compl. & 0.0 & 0.0 & 11.1 & 33.3 & 27.8 & 27.8 & 100 \\
& \% dentro de 19 & 0.0 & 0.0 & 4.3 & 12.2 & 17.2 & 16.7 & 8.9 \\
& \% del total & 0.0 & 0.0 & 1 & 3 & 2.5 & 2.5 & 8.9 \\
\hline \multirow{5}{*}{ Normal } & Recuento & 4 & 24 & 37 & 30 & 23 & 25 & 143 \\
& \% dentro de Compl. & 2.8 & 16.8 & 25.9 & 21 & 16.1 & 17.5 & 100 \\
& \% dentro de 19 & 30.8 & 68.6 & 78.7 & 61.2 & 79.3 & 83.3 & 70.4 \\
& \% del total & 2 & 11.8 & 18.2 & 14.8 & 11.3 & 12.3 & 70.4 \\
\hline \multirow{5}{*}{ Sobrepeso } & \% dentro de Compl. & 21.4 & 26.2 & 19 & 13 & 1 & 0 & 42 \\
& \%ecuento & 69.2 & 31.4 & 17 & 26.5 & 3.4 & 0.0 & 100 \\
& \% dentro de 19 total & 4.4 & 5.4 & 3.9 & 6.4 & 0.5 & 0.0 & 20.7 \\
\hline \multirow{3}{*}{ Total } & Recuento & 13 & 35 & 47 & 49 & 29 & 30 & 203 \\
& \% dentro de Compl. & 6.4 & 17.2 & 23.2 & 24.1 & 14.3 & 14.8 & 100 \\
& \% dentro de 19 & 100 & 100 & 100 & 100 & 100 & 100 & 100 \\
& \% del total & 6.4 & 17.2 & 23.2 & 24.1 & 14.3 & 14.8 & 100 \\
\hline
\end{tabular}

Fuente: elaboración propia. 
común" -, que el estar gordo es "algo equivocado" $-\mathrm{o}$, lo que es lo mismo, algo que el sujeto debería tratar de corregir-. El dato más significativo y concluyente, a este respecto, es el que indica que el $88.1 \%$ de las personas con sobrepeso entrevistadas contestaron nada a la pregunta 7 , un porcentaje que se sitúa más de 60 puntos porcentuales por encima de las cifras de los encuestados de complexión delgada o normal (Tabla 4). Parece que las personas con sobrepeso no aceptan el calificativo "equivocado" para su situación, lo que sugiere que en una buena parte tienen interiorizada la idea de que es algo respecto a lo cual su propia voluntad o actos se encuentran muy limitados.

Los datos de la encuesta pueden ser considerados ilustrativos de cómo los individuos transmiten en una buena medida sus propias percepciones personales a lo que consideran que es la percepción colectiva. Ello coincidiría con la idea, comúnmente admitida, de que en las edades juveniles el cuerpo adopta una mayor relevancia como medio de relación social; es, sin duda, en esos tramos generacionales en los que el cuidado del cuerpo y su consideración como forma de atracción social son especialmente relevantes, como se veía al considerar los análisis de Ciudad (2001). Esa traslación de la percepción individual a lo que se considera propio del conjunto de la sociedad puede apreciarse de dos maneras. De un lado, se puede considerar como muy sintomático que, como recoge la Tabla 5 , ninguno de los 39 encuestados de más de 30 años de edad seleccione la opción mucho, y solo un 12.8 la opción bastante, al responder acerca de si considera que la gente busca el cuerpo perfecto para no ser centro de crítica social. De otro lado, en la Tabla 6 se observa cómo son los encuestados de menos de 30 años los que, en una medida mucho mayor (48.2), contesta bastante o mucho a la cuestión de si ven a la sociedad como consumidora de variantes de actividad física como ropa, cirugía plástica, etc. Los datos, referentes a la misma cuestión, tabulados por género permiten apreciar (Tabla 6) cómo en el caso de los varones resultan mayoritarias las opciones bastante o mucho (57.6), mientras que en el caso de las féminas el porcentaje de ambas categorías de respuesta es mucho más reducido (28.8). Estos dígitos parecen sugerir que las mujeres contemplan ese tipo de actividades de una manera más natural, mientras que en el caso del sexo opuesto se conciben con más facilidad como el resultado del juego de interacciones sociales.

Los dígitos pueden, en este sentido, mostrar alguna paradoja, dado que aparece asumida la realidad de que existe un condicionante social en aspectos relativos al cuerpo como los señalados, pero a la vez el entrevistado individual se muestra reticente a aceptar que él o ella, personalmente, pueda sentir "algún tipo de rechazo" hacia las personas cuyo cuerpo se aparta de la norma social. Los datos de la Tabla 7 permiten apreciar cómo las opciones de respuesta bastante o mucho no cuentan con una sola elección, mientras que resulta muy ampliamente elegida la opción nada. La formulación de la pregunta pretendía, deliberadamente, recoger la expresión "algún tipo de rechazo" para que, cualquiera que fuera el grado o la forma del mismo, el entrevistado pudiera verse reflejado con facilidad; cualquier tipo de rechazo podría ser, por ejemplo, el desagrado estético que pudiera generar un individuo con problemas de obesidad. Podría concluirse que, si bien se reconoce que, en conjunto, pueda darse un rechazo social, el entrevistado tiende a expresar que se produce sin su concurso. En términos metodológicos, cabría pensar muy razonablemente que en la pregunta 5 del cuestionario los encuestados responden en función de lo que se ha dado en llamar "conformidad con las pautas de deseabilidad social", de tal manera que contestan de una manera que no suscite rechazo o que se considere más correcta o aceptable en función de las pautas de comportamiento y morales comúnmente asumidas.

\section{La dieta como instrumento de} cuidado de la imagen corporal

En todo caso, la percepción acerca de si, como consecuencia de de sus problemas y para evitar la presión social, las personas con sobrepeso se ven forzadas a seguir una dieta (ítem 8), aparece graduada en función de la complexión de los encuestados. Así, en la Tabla 8 se puede apreciar cómo a medida que se va pasando de la condición de delgado a la de 
normal y sobrepeso, los porcentajes de elecciones en muy poco o nada se amplían muy significativamente. De hecho, ninguno de los encuestados de complexión delgada elige ninguna de ambas opciones, por un 26.2 y 21.4 en cada una de ellas, respectivamente, en el caso de los encuestados con sobrepeso. Se podría pensar -en función de los dígitos- en una cierta dificultad, por parte de los individuos sin sobrepeso, para tener una comprensión real de la dificultad para evitar ese problema de las personas con disposición a padecerlo. Su solución no es, con muchísima frecuencia, solo cuestión de una dieta más o menos adecuada. Por el contrario, en las opciones bastante y mucho los porcentajes son decrecientes a medida que se avanza en la complexión corporal de los entrevistados. Las interpretaciones pueden ser diversas, pero es muy razonable considerar que un cierto número de personas con sobrepeso puede tender a pensar que deberían hacer un mayor esfuerzo de control de su alimentación (en la línea de lo que se veía en la Tabla 1), o que, en cualquier caso, un porcentaje de ellas pueden rechazar la idea de que su actitud al respecto pueda derivarse de la observación de otros.

Las percepciones y sensibilidades acerca del cuerpo, de esta manera, aparecen diferenciadas en función de características que, en sí mismas, son también corporales -como la complexión, el género o la edad-. Los dígitos que se acaban de repasar, dentro de la frialdad que inevitablemente caracteriza a la expresión numérica, revelan que cuerpo y sociedad operan de forma indisociable.

\section{Discusión y conclusión}

Los datos son ilustrativos de cómo el mundo social tiene incidencia sobre nuestro cuerpo. El uso y la percepción que se tiene de él no han aparecido al margen de la propia condición corporal, por el contrario, se puede apreciar que tres rasgos corporales básicos fundamentan matices y diferencias sustantivas en la forma de relacionarnos con el cuerpo. A estos efectos, el tener o no sobrepeso, el estar o no en el tramo de edad juvenil y el ser hombre o mujer son rasgos que condicionan la percepción que se tiene del cuerpo, a la vez que la percepción que los demás tienen de él. El cuerpo ha quedado definido como punto de conexión útil para la conformación de un mundo social.

En definitiva, tras analizar los resultados, se ha llegado a la conclusión de que, en la población objeto de estudio, coinciden en una serie de aspectos pero difieren en otros. En ocasiones las personas se sienten influenciadas ante la imagen corporal y piensan que la gente obesa está bastante estigmatizada en la sociedad actual. Creen que la gente con sobrepeso a veces se siente juzgada las 24 horas del día por el hecho de padecer una obesidad. Piensan que el resto de las personas cuidan bastante su imagen corporal por el hecho de que saben que el resto del mundo les mira, y también están de acuerdo en que son las mujeres las más afectadas por ese hecho. Piensan que puede tener una implicación para la salud el hecho de tener un cuerpo idealizado con el abuso de un determinado comportamiento y están seguros de que existe una industria del cuerpo perfecto que se beneficia económicamente de este hecho. Muy usualmente ven a la sociedad como consumidora de las variantes de la actividad física (cirugía plástica, suplementos) y piensan que puede existir una relación económica notoria ante tal consumo. No expresan un rechazo hacia las personas cuyo cuerpo se aparta de la norma social. Y además creen que alguna vez las personas con sobrepeso se obligan a seguir una dieta equilibrada para intentar superar sus problemas y evitar que la gente les vigile.

\section{Referencias}

Bourdieu, P. (1998). La distinción: criterio y bases sociales del gusto. Madrid: Taurus.

Ciudad, A. (2001, octubre). Sexo, móviles y revistas de chicas. Trabajo presentado en el VI Encuentro de Sociólogos y Politólogos en Castilla-La Mancha, Almagro (Ciudad Real), España.

Connell, R. (1995). Masculinities. Cambridge: Polity.

Crossley, N. (1995). Merleau-Ponty, the elusive body and carnal sociology. Body $\mathcal{E}$ Society, 1(1), 43-63.

Díaz Rojo, J. A. (2006). El culto a la salud y a la belleza. Madrid: Biblioteca Nueva. 
Duncan, M. C. (2007). Bodies in motion: The sociology of physical activity. Quest, 59(1), 55-66.

Freud, S. (1961). Civilization and its discontents. New York: Norton.

Giddens, A. (1984). The constitution of society. Oxford: Polity Press.

Goffman, E. (1959). The presentation of self in everyday life. New York: Doubleday.

Goffman, E. (1961). Asylums. New York: Doubleday.

Goffman, E. (1963). Behavior in public places. New York: Free Press.

Goffman, E. (1979). Gender advertisements. Cambridge, MA: Harvard University Press.

Husserl, E. (1997). Ideas II. Libro Segundo: Investigaciones fenomenológicas sobre la constitución (A. Zirión, Trad., 1a. ed.). México: UNAM, Instituto de Investigaciones Filosóficas.

Jones, M. (2008). Makeover culture's dark side: Breasts, death and Lolo Ferrari. Body $\mathcal{E}$ Society, 14(1), 89-104.

Merleau-Ponty, M. (2003). El mundo de la percepción. Siete conferencias (1a. ed.). Buenos Aires: Fondo de Cultura Económica.

Mauss, M. (1973). Techniques of the body. Economy and Society, 2(1), 70-88.

Miranda, T. (2002). El cuerpo, fuente de significado. En C. Blanco, A. Miñambres \& T. Miranda (Coords.), Pensando el cuerpo, pensando desde un cuerpo (pp. 73-83). Albacete: Universidad de Castilla-La Mancha.

Nash, R. (1999). Bourdieu, "Habitus", and educational research: Is it all worth the candle? British Journal of Sociology of Education, 20(2), 175-187.
Ruiz Calvete, M. (2010). El cuerpo humano como objeto estético. A Parte Rei. Revista de Filosofía, 72, 1-7.

Runge, A. K. (2004). Materialización del cuerpo y performatividad del género. Judith Butler y el giro a lo preformativo-discursivo en el debate sexogénero. En Memorias. Simposio Internacional Cuerpo, Motricidad y Desarrollo Humano. Medellín: Universidad de Antioquia, Instituto Universitario de Educación Física.

Rysst, M. (2010). "Healthism" and looking good: Body ideals and body practices in Norway. Scandinavian Journal of Public Health, 38(Suppl. 5), 71-80.

Salinas, L. (1994). La construcción social del cuerpo. Reis. Revista Española de Investigaciones Sociológicas, 68, 85-96.

San Martín, J. (2010). El contenido del cuerpo. Investigaciones Fenomenológicas, (Monográfico 2: Cuerpo y alteridad), 170-187.

Sánchez-Vallejo, M. A. (2009, 6 de julio). El mensaje está en el cuerpo. El País, p. 32.

Scarry, E. (1985). The body in pain: The making and unmaking of the world. Oxford: Oxford University Press.

Schutz, A. (1967). Collected Papers. The problem of social reality (Vol. 1). The Hague: Martinus Nijhoff.

Shilling, C. (1991). Educating the body: Physical capital and the production of social inequalities. Sociology, 21(4), 653-672.

Todes, S. (2001). Body and world. Cambridge, MA: MIT Press. 